\title{
A Markov process model of ocean sediments
}

\author{
Michael Sockell, loannis Besieris, and Werner Kohler \\ Virginia Polytechnic Institute and State University, Blacksburg, Virginia 24061 \\ Herbert Freese \\ Arete' Associates, P. O. Box 2951, Arlington, Virginia 22202
}

(Received 19 March 1984; accepted for publication 31 July 1984)

\begin{abstract}
Monochromatic plane-wave illumination of a randomly stratified, laterally homogeneous sediment layer is considered. The deposition process creating the stochastic layering is assumed to be a continuous parameter, finite state Markov chain. A Riccati equation for the plane-wave reflection coefficient is formulated and first-order partial differential equations for relevant probability density functions are subsequently obtained. These equations are solved numerically for a two-material turbidite model similar to the one considered by Gilbert [J. Acoust. Soc. Am. 68, 1454-1458 (1980)]. Statistical moments of the reflection coefficient are computed at 25 and $250 \mathrm{~Hz}$ as a function of overall sediment thickness. These equations are also used to derive the nonrandom or "smooth" geoacoustic model that is appropriate in the low-frequency limit.
\end{abstract}

PACS numbers: 43.30.Bp, 43.30.Dr, 91.50.Jc, 92.10.Vz

\section{INTRODUCTION}

We consider a transversely homogeneous, randomly stratified ocean sediment model. The sediment layer is assumed to rest upon a homogeneous, semi-infinite rock basement and to lie beneath an isovelocity water column. A stochastic model is adopted because the layering fine structure within the sediment cannot be accurately accounted for; we thus opt for a cruder but more tractable point of view in which the composition and resulting acoustic behavior of the medium is described probabilistically. The particular deposition process that created the sediment layer is assumed to be a continuous parameter, finite state Markov chain. This model, which is qualitatively similar to the one adopted by Gilbert $^{1}$ in his simulations, leads to a straightforward analytic description; the model is constructed in the following way. We assume that $\boldsymbol{N}$ different materials (various types of clay, sand, silt, etc.), each having its own characteristic density and sound speed, enter into the formation of the overall sediment layer. Initially, at the rock-sediment interface, each of the $N$ materials (states) can be present with a certain prescribed probability (perhaps equally likely). Then, as the sediment thickness increases, the composition of the sediment switches randomly among the $N$ constituent materials according to the dictates of a prescribed transition mechanism. This transition law (which would be empirically determined, typically by core sampling) can be made nonstationary (i.e., variable) to account for variations in layering statistics with depth. A slowly varying gradient is added to the model to account for compactification-induced refractive effects. The sediment model considered, therefore, is a random multilayer (slowly nonstationary $N$-state Markov chain) superimposed upon a slowly varying refracting gradient. The Markovian hypothesis, ${ }^{2}$ in which the future evolution of the deposition process is assumed conditionally independent of its past history, is a reasonable idealization. This sediment model is more fully discussed in Sec. II.

We assume that a monochromatic plane wave is incident upon the sediment from the water column. The quanti- ties of interest are the statistics of the plane-wave reflection coefficient [defined in Eqs. (4)]; from this information, the statistics of the reflected signal in the water column (e.g., the two-point mutual coherence function for the complex pressure) can be readily inferred. The Markov chain assumption permits us to formulate exact equations for the relevant probability density functions; the solution of these equations provides all the desired statistical information.

In Sec. I we formulate the original stochastic two-point boundary value problem of interest and recast it as an initial value problem (Riccati equation and initial condition) for the reflection coefficient. In Sec. II, following the exposition of Morrison, ${ }^{3}$ we obtain first-order partial differential equations for probability density functions characterizing the reflection coefficient statistics. A turbidite model is analyzed in Sec. III by numerically solving these equations. Section IV presents our conclusions.

\section{PROBLEM FORMULATION}

The problem that we consider is sketched in Fig. 1. A sediment layer, lying upon a homogeneous, semi-infinite rock basement, is illuminated by a monochromatic plane wave from an isovelocity water column. The basic equations for the pressure and particle velocity are ${ }^{4}$

$$
\partial_{t} p+\rho c^{2} \nabla \cdot v=0, \quad \partial_{t} \mathbf{v}+\rho^{-1} \nabla p=0,
$$

where the density $\rho$ and the sound speed $c$ are modeled as piecewise-constant random functions of depth $z$ within the sediment layer. Because of the assumed transverse homogeneity and plane-wave excitation, Eqs. (1) ultimately reduce to a linear system of ordinary differential equations for the problem of interest. Let the pressure $p$ and $z$ component of particle velocity $v_{z}$ be represented as

$$
\begin{aligned}
& p(x, z, t) \equiv \hat{p}(z) \exp \left[i\left(k x \sin \theta_{0}-\omega t\right)\right], \\
& v_{z}(x, z, t) \equiv\left(\rho_{0} c_{0}\right)^{-1} \hat{v}_{z}(z) \exp \left[i\left(k x \sin \theta_{0}-\omega t\right)\right],
\end{aligned}
$$

where $\omega$ denotes radian frequency, $k=\omega / c_{0}$ is wavenumber referenced to the sound speed of the water, and $\theta_{0}$ is the 


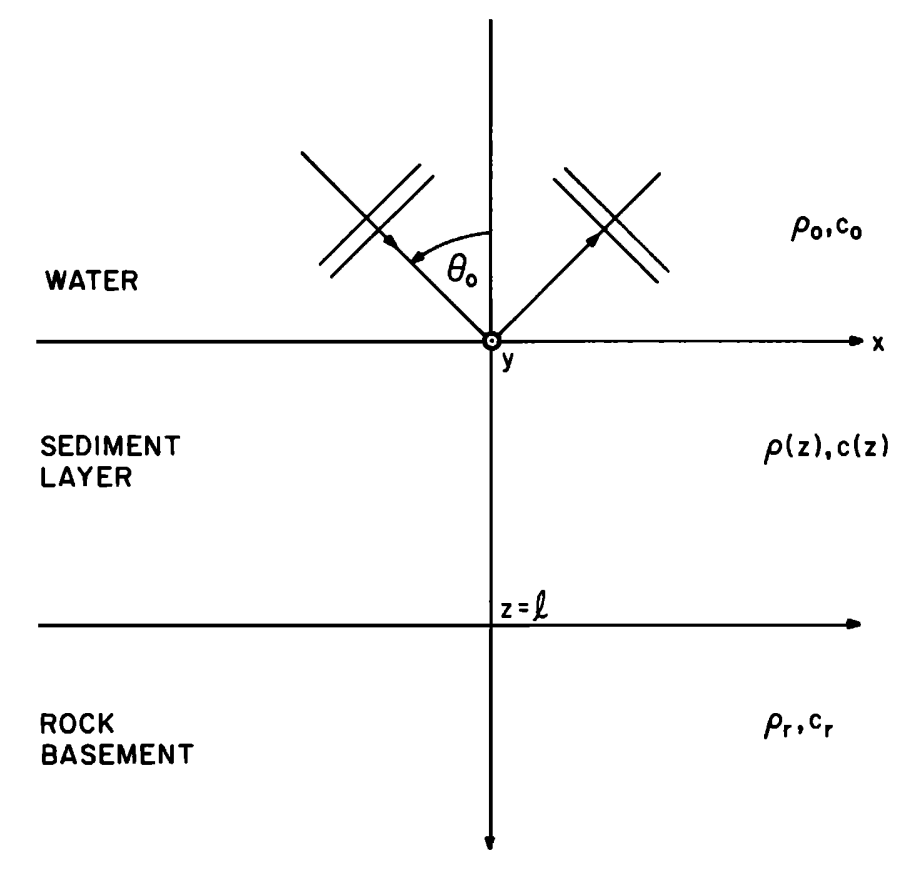

FIG. 1. Problem configuration.

incidence angle measured from normal (cf. Fig. 1). For brevity, let $\tilde{\rho} \equiv \rho / \rho_{0}$ and $\tilde{c} \equiv c / c_{0}$. Then, the substitution of (2) into (1) leads to the following first-order system of equations:

$$
\frac{d}{d z}\left[\begin{array}{l}
\hat{p} \\
\hat{v}_{z}
\end{array}\right]=i k\left[\begin{array}{cc}
0 & \tilde{\rho} \\
\tilde{\rho}^{-1}\left(\tilde{c}^{2}-\sin ^{2} \theta_{0}\right) & 0
\end{array}\right]\left[\begin{array}{l}
\hat{p} \\
\hat{v}_{z}
\end{array}\right] .
$$

The basic problem involving Eqs. (3) is a stochastic twopoint boundary value problem. [Equations (7) will clearly establish this point.] In the water column $(z<0)$, where $\tilde{\rho}$ and $\tilde{c}$ are both unity, a unit amplitude excitation leads to a solution of the form:

$$
\begin{aligned}
& \hat{p}(z)=\exp \left(i k z \cos \theta_{0}\right)+R \exp \left(-i k z \cos \theta_{0}\right), \\
& \hat{v}_{z}(z)=\cos \theta_{0}\left[\exp \left(i k z \cos \theta_{0}\right)-R \exp \left(-i k z \cos \theta_{0}\right)\right],
\end{aligned}
$$

where the complex constant $R$ is the unknown reflection coefficient whose statistical properties constitute the goals of this study. Within the sediment layer $(0 \leqslant z \leqslant l), \tilde{\rho}$ and $\tilde{c}$ are random functions of depth $z$. In the rock basement $(l<z<\infty)$, imposition of the downward-propagating radiation condition leads to the solution:

$$
\begin{aligned}
\hat{p}(z)= & t \exp \left[i k\left(\tilde{c}_{r}^{-2}-\sin ^{2} \theta_{0}\right)^{1 / 2}(z-l)\right], \\
\hat{v}_{z}(z)= & \tilde{\rho}_{r}^{-1}\left(\tilde{c}_{r}^{-2}-\sin ^{2} \theta_{0}\right)^{1 / 2} t \\
& \times \exp \left[i k\left(\tilde{c}_{r}^{-2}-\sin ^{2} \theta_{0}\right)^{1 / 2}(z-l)\right],
\end{aligned}
$$

where $\tilde{\rho}_{r} \equiv \rho_{r} / \rho_{0}, \tilde{c}_{r} \equiv c_{r} / c_{0}$, and $t=p(l)$ is an unknown transmission coefficient. Specification of the boundary value problem is completed by requiring that $\hat{p}$ and $\hat{v}_{z}$ be continuous across the interfaces at $z=0$ and $z=l$.

Our immediate goal is to recast this linear stochastic boundary value problem into a nonlinear stochastic initial value problem for the reflection coefficient (with sediment thickness $l$ serving as independent variable). This step is crucial to the probabilistic analysis because stochastic initial value problems possess a causal, evolutionary structure not shared by boundary value problems. This step will be accomplished in two stages. We first introduce new dependent var- iables, scattering variables $a$ and $b$ defined by

$$
\begin{aligned}
& a(z) \equiv \frac{1}{2}\left[\hat{p}(z)+\hat{v}_{z}(z) \sec \theta_{0}\right], \\
& b(z) \equiv \frac{1}{2}\left[\hat{p}(z)-\hat{v}_{z}(z) \sec \theta_{0}\right] .
\end{aligned}
$$

In terms of these new dependent variables, the boundary value problem of interest becomes

$$
\begin{aligned}
& \frac{d}{d z}\left[\begin{array}{l}
a \\
b
\end{array}\right]=\frac{i k}{2}\left[\begin{array}{cc}
a_{11} & a_{12} \\
-a_{12} & -a_{11}
\end{array}\right]\left[\begin{array}{l}
a \\
b
\end{array}\right], \quad 0<z<l, \\
& a_{11} \equiv \tilde{\rho} \cos \theta_{0}+\left(\tilde{\rho} \cos \theta_{0}\right)^{-1}\left(\tilde{c}^{2}-\sin ^{2} \theta_{0}\right), \\
& a_{12} \equiv-\tilde{\rho} \cos \theta_{0}+\left(\tilde{\rho} \cos \theta_{0}\right)^{-1}\left(\tilde{c}^{2}-\sin ^{2} \theta_{0}\right), \\
& a(0)=1, \quad b(0)=R, \\
& b(l)=\Gamma_{r} a(l), \\
& \Gamma_{r} \equiv\left[\tilde{\rho}_{r} \cos \theta_{0}-\left(\tilde{c}_{r}^{-2}-\sin ^{2} \theta_{0}\right)^{1 / 2}\right] \\
& \quad \times\left[\tilde{\rho}_{r} \cos \theta_{0}+\left(\tilde{c}_{r}^{-2}-\sin ^{2} \theta_{0}\right)^{1 / 2}\right]^{-1}, \\
& a, b \operatorname{continuous~across} z=0 \text { and } z=l .
\end{aligned}
$$

Thus, $a$ represents a downward-propagating incident wave while $b$ is an upward-propagating reflected wave. In particular, the reflection coefficient $R$ is determined by evaluating the ratio $b / a$ at the water-sediment interface $(z=0)$.

To obtain the Riccati equation for the problem, it proves convenient to relocate the coordinate system origin at the sediment-rock interface. We introduce the new independent variable $\xi \equiv l-z$ and define $\hat{R}(\xi) \equiv b / a$. One can show, by direct calculation using (7), that

$$
\begin{aligned}
& \frac{d \hat{R}}{d \xi}=i k a_{11} \hat{R}+i \frac{k}{2} a_{12}\left(1+\hat{R}^{2}\right), \quad 0<\xi<l, \\
& \hat{R}(0)=\Gamma_{r},
\end{aligned}
$$

where $a_{11}$ and $a_{12}$ are viewed as functions of $\xi$. The linear two-point boundary value problem (7) has thus been replaced by a nonlinear initial value problem (8). We must solve (8) and determine $\hat{R}(l)$, which is the desired reflection coefficient, i.e., $\hat{R}(l)=R$.

\section{STOCHASTIC ANALYSIS}

Problem (8) has the advantage of being a stochastic initial value problem. Recall that in the sediment layer, $\tilde{\rho}$ and $\tilde{c}$ (and hence $a_{11}$ and $a_{12}$ ) switch values randomly among $N$ states (materials) as distance from the rock interface increases. In contrast to boundary value problems, the evolutionary or causal nature of initial value problems leads to a relatively straightforward probabilistic characterization.

As a first step, we shall introduce polar coordinates; let

$$
\hat{\boldsymbol{R}}(\xi) \equiv r(\xi) e^{i \theta(\xi)}, \quad \Gamma_{r} \equiv \gamma_{r} e^{i \phi_{r}} .
$$

In terms of these polar variables, problem (8) becomes

$$
\begin{aligned}
& \frac{d r}{d \xi}=\frac{k}{2} a_{12}\left(1-r^{2}\right) \sin \theta, \quad r(0)=\gamma_{r}, \\
& \frac{d \theta}{d \xi}=\frac{k}{2} a_{12}\left(r^{-1}+r\right) \cos \theta+k a_{11}, \quad \theta(0)=\phi_{r} .
\end{aligned}
$$

From (10) one can show that $r(\xi) \leqslant 1$ (since $\left.0 \leqslant \gamma_{r} \leqslant 1\right)$ and that $r(\xi) \equiv 1$ if $\gamma_{r}=1$. (For simplicity, no dissipation has as yet been incorporated into the sediment model.)

Let $\tau(\cdot)$ denote the underlying continuous parameter $N$ - 
state Markov chain that controls the random sedimentary deposition process. ${ }^{5}$ Thus, if $\tau(\xi)=n$ for a given random sediment realization, then for that particular realization the $n$th material is presented at distance $\xi$ from the rock basement. As Morrison ${ }^{3}$ has observed, the joint process $[r(\xi)$, $\theta(\xi), \tau(\xi)]$ is a Markov process. First-order partial differential equations describe the evolution of its probabilistic structure; we must introduce some additional notation, however, in order to state these equations.

Weshall let $Q(\xi) \equiv\left[q_{m n}(\xi)\right]$ denotethe $N \times N$ transition matrix for the $\tau(\cdot)$ process. Thus, we assume that

$$
\begin{aligned}
& q_{m n}(\xi) \geqslant 0, \quad m \neq n, \quad q_{m m}(\xi) \leqslant 0, \quad m, n=1,2, \ldots, N \\
& \sum_{n=1}^{N} q_{m n}(\xi)=0, \quad m=1,2, \ldots, N .
\end{aligned}
$$

This transition matrix, together with the initial probabilities (at the rock basement), defines the probabilistic structure of the sediment deposition process. Loosely speaking:

(1) The probability that the sediment state (material) does not change over the interval $(\xi, \xi+\delta \xi)$, given that it initially was in state $n$ is $^{6}: 1+q_{n n}(\xi) \delta \xi+o(\delta \xi)$.

(2) The probability that the sediment layering changes precisely once over the interval $(\xi, \xi+\delta \xi)$ to state $n$, given that it initially was in state $m$ is: $q_{m n}(\xi) \delta \xi+o(\delta \xi)$.

(3) The probability that the sediment layering undergoes more than one change of state over the interval $(\xi$, $\xi+\delta \xi)$ is: $o(\delta \xi)$.

A precise statement is given by Morrison. ${ }^{3}$ Note that the summation equation in (11) is simply a statement of conservation of probability. The transition matrix must be determined empirically, typically by studying the layering structure in core samples. Information about material types, the relative frequency of their occurrence in the layering, layer thicknesses, layering patterns, etc., is all contained within this matrix. Large scale variations in layering statistics can be accounted for by making the transition matrix nonstationary, i.e., a slowly varying function of $\xi$; if the layering statistics are stationary, $Q$ will be a constant matrix. [Consider, for example, a hypothetical case in which material 1 is a clay while material 3 is a silty sand. Then, given that clay is present at height $\xi$ above the basement, the probability [apart from $o(\delta \xi)$ terms] that clay will remain present over the $(\xi, \xi+\delta \xi)$ interval is $1+q_{11}(\xi) \delta \xi=1-\left|q_{11}(\xi)\right| \delta \xi$ while the probability that the sediment will switch to silty sand in the interval is $q_{13}(\xi) d \xi$.) The remaining information that must be prescribed in order to completely specify the stochastic description of the sediment deposition process is the set of initial probabilities, the probabilities of finding each of the sediment materials at the rock basement interface. We define

$$
\begin{aligned}
& \pi_{n} \equiv P\{\tau(0)=n\}, \quad n=1,2, \ldots, N, \\
& \pi_{n} \geqslant 0, \quad \sum_{n=1}^{N} \pi_{n}=1 .
\end{aligned}
$$

With the sediment layering statistics characterized by transition matrix $Q$ and initial probabilities $\left\{\pi_{n}\right\}_{n=1}^{N}$ and the reflection coefficient dynamics specified by (8), we are in a position to describe the probabilistic evolution of the joint process $[r(\xi), \theta(\xi), \tau(\xi)]$. Let

$$
\begin{gathered}
p_{n}(r, \theta, \xi) d r d \theta \equiv P\{r \leqslant r(\xi) \leqslant r+d r, \theta \leqslant \theta(\xi) \leqslant \theta+d \theta, \\
\tau(\xi)=n\}, \quad n=1,2, \ldots, N .
\end{gathered}
$$

Then, as a special case of the derivation presented by Morrison, ${ }^{3}$ one can show that the probability density functions $\left\{p_{n}\right\}_{n=1}^{N}$ are solutions of the following initial value problem:

$$
\begin{aligned}
& \partial_{\xi} p_{n}+\partial_{r}\left[(k / 2) a_{12}^{(n)}\left(1-r^{2}\right) \sin \theta p_{n}\right] \\
& \quad+\partial_{\theta}\left\{\left[(k / 2) a_{12}^{(n)}\left(r^{-1}+r\right) \cos \theta+k a_{11}^{(n)}\right] p_{n}\right\} \\
& \quad-\sum_{m=1}^{N} q_{m n} p_{m}=0, \quad n=1,2, \ldots, N, \\
& p_{n}(r, \theta, 0)=\pi_{n} \delta\left(r-\gamma_{r}\right) \delta\left(\theta-\phi_{r}\right),
\end{aligned}
$$

where $a_{11}^{(n)}$ and $a_{12}^{(n)}$ refer to $a_{11}$ and $a_{12}$ defined in (7) with the random portions of the normalized density and sound speed set equal to the values associated with the $n$th material. The initial conditions are delta functions since the rock basement is assumed to be nonrandom, i.e., $r$ and $\theta$ are known with certitude to be $\gamma_{r}$ and $\phi_{r}$, respectively. To determine the expected value of a function of $r(\xi), \theta(\xi)$, say $f[r(\xi), \theta(\xi)]$, we must solve initial value problem (14) and compute:

$$
E\{f[r(\xi), \theta(\xi)]\}=\sum_{n=1}^{N} \int_{0}^{2 \pi} \int_{0}^{1} f(r, \theta) p_{n}(r, \theta, \xi) d r d \theta .
$$

We shall introduce dissipation into the model by making the sound speed velocity complex, ${ }^{7}$ i.e., $\tilde{c} \rightarrow \tilde{c}-i \tilde{c}^{\prime}$ (with $\left.\tilde{c}^{\prime}<\tilde{c}\right)$. Noting (7), this modification is tantamount to making the changes $a_{11} \rightarrow a_{11}+i \delta, a_{12} \rightarrow a_{12}+i \delta$, with $\delta \simeq 2 \tilde{c}^{\prime} /$ $\left(\tilde{\rho} \tilde{c}^{3} \cos \theta_{0}\right)$. Equations (10) then transform to

$$
\begin{aligned}
& \frac{d r}{d \xi}=-k \delta r-\frac{k}{2} \delta \cos \theta\left(1+r^{2}\right)+\frac{k}{2} a_{12} \sin \theta\left(1-r^{2}\right), \\
& \frac{d \theta}{d \xi}=\frac{k}{2} \delta \sin \theta\left(r^{-1}-r\right)+\frac{k}{2} a_{12} \cos \theta\left(r^{-1}+r\right)+k a_{11},
\end{aligned}
$$

while Eqs. (14) become

$$
\begin{aligned}
\partial_{\xi} p_{n} & +\partial_{r}\left\{\left[-k \delta^{(n)} r-(k / 2) \delta^{(n)} \cos \theta\left(1+r^{2}\right)\right.\right. \\
& \left.\left.+(k / 2) a_{12}^{(n)}\left(1-r^{2}\right) \sin \theta\right] p_{n}\right\} \\
& +\partial_{\theta}\left\{\left[(k / 2) \delta^{(n)} \sin \theta\left(r^{-1}-r\right)\right.\right. \\
& \left.\left.+(k / 2) a_{12}^{(n)}\left(r^{-1}+r\right) \cos \theta+k a_{11}^{(n)}\right] p_{n}\right\} \\
& -\sum_{m=1}^{N} q_{m n} p_{m}=0
\end{aligned}
$$

where $\delta^{(n)}$ represents the loss coefficient for the $n$th material.

\section{A. Remark}

In certain applications, it may be desirable to describe the process in terms of Cartesian rather than polar coordinates. If, instead of (9), we let

$$
\hat{R}(\xi) \equiv x(\xi)+i y(\xi)
$$

and define

$$
\begin{aligned}
& \hat{p}_{n}(x, y, \xi) d x d y \\
& \quad \equiv P\{x<x(\xi)<x+d x, y<y(\xi)<y+d y, \tau(\xi)=n\}, \\
& n=1,2, \ldots, N,
\end{aligned}
$$


we obtain the following initial value problem for $\left\{\hat{p}_{n}\right\}_{n=1}^{N}$ :

$$
\begin{aligned}
\partial_{\xi} \hat{p}_{n} & +\partial_{x}\left\{\left[-k \delta^{(n)} x-(k / 2) \delta^{(n)}\left(1+x^{2}+y^{2}\right)-k a_{11}^{(n)} y\right.\right. \\
& \left.\left.-k a_{12}^{(n)} x y\right] \hat{p}_{n}\right\}+\partial_{y}\left\{\left[-k \delta^{(n)} y-k \delta^{(n)} x y+k a_{11}^{(n)} x\right.\right. \\
& \left.\left.+(k / 2) a_{12}^{(n)}\left(1+x^{2}-y^{2}\right)\right] \hat{p}_{n}\right\}-\sum_{m=1}^{N} q_{m n} \hat{p}_{m}=0,
\end{aligned}
$$

$0 \leqslant x^{2}+y^{2} \leqslant 1, \quad n=1,2, \ldots, N$,

$\hat{p}_{n}(x, y, 0)=\pi_{n} \delta\left(x-\gamma_{r} \cos \phi_{r}\right) \delta\left(y-\gamma_{r} \sin \phi_{r}\right)$.

In this case, the expected value of a function of $x(\xi), y(\xi)$ \{ call it $g[x(\xi), y(\xi)]\}$ is determined by

$$
\begin{aligned}
& E\{g[x(\xi), y(\xi)]\} \\
& \quad=\sum_{n=1}^{N} \iint_{x^{2}+y^{2}<1} g(x, y) \hat{p}_{n}(x, y, \xi) d x d y .
\end{aligned}
$$

In particular, the well-behavedness of the coefficients in (20) makes the Cartesian coordinate formulation attractive for numerical implementation.

\section{APPLICATION TO A TURBIDITE MODEL}

We shall apply the theory developed in Secs. I and II to a two-material $(N=2)$ turbidite model similar to the one considered by Gilbert. ${ }^{1}$ For simplicity we assume stationary layering statistics so that transition matrix $Q$ is a constant $2 \times 2$ matrix. As shown by Doob, ${ }^{8}$ the probability density function for the layer thickness of the $n$th material (call it $L_{n}$ ) is

$$
\operatorname{Pr}\left(l \leqslant L_{n} \leqslant l+d l\right)=-q_{n n} e^{q_{n n} l} d l, \quad 0 \leqslant l<\infty .
$$

From (11) we note that the transition matrix $Q$ is completely determined (in the two-material case) by a specification of the average layer thicknesses. Let $\left\langle L_{n}\right\rangle$ denote the mean or average layer thickness for the $n$th material. Then, (11) and (22) imply that

$$
Q=\left[\begin{array}{cc}
-\left\langle L_{1}\right\rangle^{-1} & \left\langle L_{1}\right\rangle^{-1} \\
\left\langle L_{2}\right\rangle^{-1} & -\left\langle L_{2}\right\rangle^{-1}
\end{array}\right]
$$

For this model (in contrast to the one studied by Gilbert) one cannot independently specify layer thickness fluctuations; from (22) it follows that

$$
\sigma_{n}^{2} \equiv\left\langle\left(L_{n}-\left\langle L_{n}\right\rangle\right)^{2}\right\rangle=\left\langle L_{n}\right\rangle^{2} \text {. }
$$

Equations (14) [or (20)] have the general form

$$
\partial_{\xi} \mathbf{p}+k \mathscr{L} \mathbf{p}+Q^{T} \mathbf{p}=\mathbf{0},
$$

where $\mathrm{p}=\left(p_{1} p_{2}\right)^{T}$ is the two-dimensional vector of probabilities, $\mathscr{L}$ is a (diagonal) matrix differential operator, and $Q^{T}$ is the matrix transpose of (23). Neglect for the moment the length scales associated with dissipation and refraction; then, (23) and (25) concisely exhibit the remaining two important scales of the problem. The first-order diagonal differential operator $k \mathscr{L}$ acts to convect the probability masses $p_{1}$ and $p_{2}$ around the unit disk along two independent families of characteristics (determined by the two types of material). This operator acts on a scale proportional to wavelength $\lambda$ (i.e., with a "strength" proportional to $\lambda^{-1}$ ). The matrix operator $Q^{T}$ acts to couple the components of p, i.e., to exchange probability mass between the components; this operator acts on a scale proportional to average layer thickness [the eigenvalues of $Q$ are 0 and $-\left(\left\langle L_{1}\right\rangle^{-1}+\left\langle L_{2}\right\rangle^{-1}\right)$ ].

As $\xi$ increases, therefore, the initial delta function probability masses move at different speeds around the unit disk while simultaneously "smearing out" and deforming due to the layering. The relative importance of each of these effects depends on the ratio of wavelength to average layering thickness.

Before considering a numerical solution of these equations, we consider the structure of (25) in the context of a low-frequency geoacoustic limit. It is clear that as wavelength increases, the fine layering structure becomes increasingly "averaged"; the acoustic response of the random sediment multilayer should tend toward that of a "smooth," nonrandom medium. ${ }^{1}$ To determine this limiting low-frequency characterization, we study $(25)$ in the regime wherein wavelength greatly exceeds average layer thickness. Wavenumber $k$ is then a small parameter and (25) becomes amenable to a two-scale asymptotic analysis. In the Appendix we show that the limiting low-frequency behavior of the solution to $(25)$ is

$$
\mathbf{p}(r, \theta, \xi) \sim p_{0}(r, \theta, \xi) \mathbf{u}, \quad \xi \sim O(\lambda),
$$

where

$$
\mathbf{u}=\left(\left\langle L_{1}\right\rangle+\left\langle L_{2}\right\rangle\right)^{-1}\left[\begin{array}{l}
\left\langle L_{1}\right\rangle \\
\left\langle L_{2}\right\rangle
\end{array}\right]
$$

is the (appropriately normalized) eigenvector of $Q^{T}$ having eigenvalue 0 and the scalar probability density function $p_{0}$ is a solution of the initial value problem:

$$
\begin{aligned}
& \partial_{\xi} p_{0}+k \partial_{r}\left\{\left[-\bar{\delta} r-\frac{1}{2} \bar{\delta} \cos \theta\left(1+r^{2}\right)\right.\right. \\
& \left.\left.\quad+\frac{1}{2} \bar{a}_{12}\left(1-r^{2}\right) \sin \theta\right] p_{0}\right]+k \partial_{\theta}\left\{\left[\frac{1}{2} \bar{\delta} \sin \theta\left(r^{-1}-r\right)\right.\right. \\
& \left.\left.\quad+\frac{1}{2} \bar{a}_{12}\left(r^{-1}+r\right) \cos \theta+\bar{a}_{11}\right] p_{0}\right\}=0 \\
& p_{0}(r, \theta, 0)=\delta\left(r-\gamma_{r}\right) \delta\left(\theta-\phi_{r}\right),
\end{aligned}
$$

where

$$
\begin{aligned}
& \bar{\delta} \equiv\left(\left\langle L_{1}\right\rangle \delta^{\langle 1\rangle}+\left\langle L_{2}\right\rangle \delta^{\langle 2\rangle}\right) /\left(\left\langle L_{1}\right\rangle+\left\langle L_{2}\right\rangle\right), \\
& \bar{a}_{1 j} \equiv\left(\left\langle L_{1}\right\rangle a_{1 j}^{\langle 1\rangle}+\left\langle L_{2}\right\rangle a_{1 j}^{\langle 2\rangle}\right) /\left(\left\langle L_{1}\right\rangle+\left\langle L_{2}\right\rangle\right), \\
& j=1,2 .
\end{aligned}
$$

Note that $\lim _{\xi \rightarrow \infty} e^{Q^{T} \xi_{\pi}}=\mathbf{u}$ for any initial probability vector $\pi=\left(\pi_{1} \pi_{2}\right)^{T}$ [cf. (12)]; thus u represents the limiting probability distribution for the layering process $\tau$. The physical implications of these observations can now be readily drawn. Note from Eq. (27a) that $p_{0}$ satisfies a first-order, linear, homogeneous partial differential equation with a delta function initial condition. As $\xi$ increases, therefore, the solution $p_{0}$ remains a delta function, suitably transported along the characteristics of the equation. The behavior of the medium is thus "smooth" or nonrandom since the probability remains a point mass. From (27b), we note that this low-frequency limiting behavior can be obtained directly from boundary value problem (7) by simply replacing the random matrix coefficients $a_{1 j}$ with their average values $\bar{a}_{1 j}, j=1,2$ (with dissipation suitably introduced and averaged).

In summary, then, we conclude that the low-frequency limiting behavior of this random sedimentary medium can 
be described by Eqs. (7), if we replace the random matrix coefficients by their average values, the averages being taken with respect to the limiting probability distribution of the layering statistics. It is important to observe that since density and sound speed enter into these coefficients nonlinearly, these averages are not tantamount to simply replacing density and sound speed by their respective mean values. Observations of the same general type, concerning the lowfrequency geoacoustic limit for a periodically layered acoustic half-space, have been made by Schoenberg and Sen ${ }^{9}$ and Gilbert. ${ }^{10}$

\section{A. Remark}

The ideas that we have developed can be easily extended to multifrequency statistics. In problems involving pulse propagation, the use of Fourier transforms in the study of the mutual coherence function requires a knowledge of twofrequency joint statistics. One must compute $\left\langle R(k) R^{*}\left(k^{\prime}\right)\right\rangle$, where $R(k)$ denotes the reflection coefficient at wavenumber $k$. In this case, using the fact that the joint process $[r(\xi), \theta(\xi)$, $\left.r^{\prime}(\xi), \theta^{\prime}(\xi), \tau(\xi)\right]$ is a Markov process (where $r^{\prime}$ and $\theta^{\prime}$ denote the polar coordinates at wavenumber $\left.k^{\prime}\right)$, we can derive the following extension of (25):

$$
\begin{aligned}
& \partial_{\xi} \mathbf{p}+k \mathscr{L}_{\mathbf{p}}+k^{\prime} \mathscr{L}^{\prime} \mathbf{p}-Q^{T} \mathbf{p}=\mathbf{0}, \\
& \mathbf{p}\left(r, \theta, r^{\prime}, \theta^{\prime}, 0\right)=\delta\left(r-\gamma_{r}\right) \delta\left(\theta-\phi_{r}\right) \delta\left(r^{\prime}-\gamma_{r}^{\prime}\right) \delta\left(\theta^{\prime}-\theta_{r}^{\prime}\right) \pi,
\end{aligned}
$$

where $\gamma_{r}^{\prime}$ and $\phi_{r}^{\prime}$ are the polar coordinates of the basement reflection coefficient at wavenumber $k^{\prime}$ and $\mathscr{L}^{\prime}$ is the diagonal differential operator obtained from $\mathscr{L}$ by replacing $(r, \theta)$ by $\left(r^{\prime}, \theta^{\prime}\right)$. If the same low-frequency asymptotic analysis is performed on (28) we obtain

$$
\mathbf{p}\left(r, \theta, r^{\prime}, \theta^{\prime}, \xi\right) \sim p_{0}(r, \theta, \xi) p_{0}^{\prime}\left(r^{\prime}, \theta^{\prime}, \xi\right) \mathbf{u},
$$

where $p_{0}$ and $p_{0}^{\prime}$ satisfy (27) (in the latter case $r, \theta, k, \gamma_{r}$, and $\phi_{r}$ are replaced by their primed counterparts).

\section{B. Numerical study}

The parameter values of Table I (taken from Table I of Ref. 1) were used in a numerical solution of initial value problem (20) for a thin, nonrefracting sediment layer. Note, however, that the layer thickness fluctuations [given by (24)] vary from those adopted in Ref. 1 . The results of this numeri- cal evaluation (at frequencies of 25 and $250 \mathrm{~Hz}$ and at several incidence angles) are summarized in Figs. 2-5. These figures display the variation of different moments of the solution [computed using (15) or (21)] as a function of sediment thickness. We shall now discuss their content.

Figure 2(a)-(c) displays the variation of the mean reflection coefficient $\langle R\rangle$ as a function of sediment thickness at 25 $\mathrm{Hz}$ for incidence angles of $0^{\circ}, 45^{\circ}$, and $85^{\circ}$, respectively: At any given sediment thickness, $\langle R\rangle=E\left\{r e^{i \theta}\right\}[f(r, \theta)$ $=r e^{i \theta}$ in (15)] is a point in the complex plane; as the layer thickness changes, the location of this point likewise changes. Figure 2 displays the locus of this point as sediment layer builds up in thickness from 0 to $50 \mathrm{~m}$; the points marked (a) correspond to no sediment present and represent the reflection coefficient of the semi-infinite rock basement while the points marked (b) represent the mean reflection coefficient of the basement covered by the random $50-\mathrm{m}$ sediment layer. As thickness increases, the locus of the mean reflection coefficient spirals in a counterclockwise manner slowly toward the origin. As one would expect, the rate of the spiraling or "phasewrapping" decreases as incidence angle increases (i.e., as grazing angle decreases).

Figure 3(a)-(c) displays the corresponding behavior of the mean reflection coefficient at $250 \mathrm{~Hz}$. The same general behavior as a function of incidence angle exists although the amount of "phasewrapping" is obviously much greater at this higher frequency. The relative amount of migration toward the origin, i.e., the relative attenuation of $|\langle R\rangle|$ by the sediment layer (at a given incidence angle) is also greater at 250 than at $25 \mathrm{~Hz}$. The effects of increased dissipation at the higher frequency thus outweigh any tendency of the layering fine structure to increase reflectivity; note that at $250 \mathrm{~Hz}$ the average layer thicknesses remain considerably less than a wavelength.

While Fig. 2 basically displays the behavior of the "center of probability mass" at $25 \mathrm{~Hz}$, Fig. 4(a)-(d) provides higher moment information at this frequency and thus provides some insight into how this probability mass itself is configured. In these figures, sediment thickness serves as abscissa and the results for all three incidence angles are presented simultaneously. Figure 4(a), (b), and (d) shows how the first, second, and fourth moments of the reflection coefficient modulus (i.e., $\left.E\left[r^{m}\right\}, m=1,2,4\right)$ vary with sediment thickness.

\begin{tabular}{|c|c|c|c|c|c|c|c|}
\hline \multirow[b]{2}{*}{ Medium } & \multirow{2}{*}{$\begin{array}{c}\text { Average layer } \\
\text { thickness }\langle L\rangle \\
\text { (m) }\end{array}$} & \multirow{2}{*}{$\begin{array}{l}\text { Layer thickness } \\
\text { fluctuation } \\
\sqrt{\left\langle(L-\langle L\rangle)^{2}\right\rangle} \\
\text { (m) }\end{array}$} & \multicolumn{2}{|c|}{$\begin{array}{c}\text { Velocity } \\
(\mathrm{m} / \mathrm{s})\end{array}$} & \multicolumn{2}{|c|}{$\begin{array}{c}\text { Absorption } \\
\text { (dB/m/kHz) }\end{array}$} & \multirow{2}{*}{$\begin{array}{l}\text { Density } \\
\left(\mathrm{g} / \mathrm{cm}^{3}\right)\end{array}$} \\
\hline & & & $p$ & $s$ & $p$ & $s$ & \\
\hline Water & Half-space & $\ldots$ & 1540 & 0 & 0 & 0 & 1.04 \\
\hline $\begin{array}{l}\text { Sediment: } \\
\text { Material } 1 \\
\text { Material } 2\end{array}$ & $\begin{array}{l}1.0 \\
0.2\end{array}$ & $\begin{array}{l}1.0 \\
0.2\end{array}$ & $\begin{array}{l}1517 \\
1646\end{array}$ & $\begin{array}{l}0 \\
0\end{array}$ & $\begin{array}{l}0.05 \\
0.15\end{array}$ & $\begin{array}{l}0 \\
0\end{array}$ & $\begin{array}{l}1.353 \\
1.652\end{array}$ \\
\hline Basement & Half-space & $\ldots$ & 4725 & 2341 & 0.03 & 0.12 & 2.54 \\
\hline
\end{tabular}
Figure 4(c) displays "moment of inertia" information, i.e.,

TABLE I. Parameter values for numerical study.

Each sediment material was assumed equally likely to exist at the basement-sediment interface, i.e., $\pi_{1}=\pi_{2}=\frac{1}{2}$. 

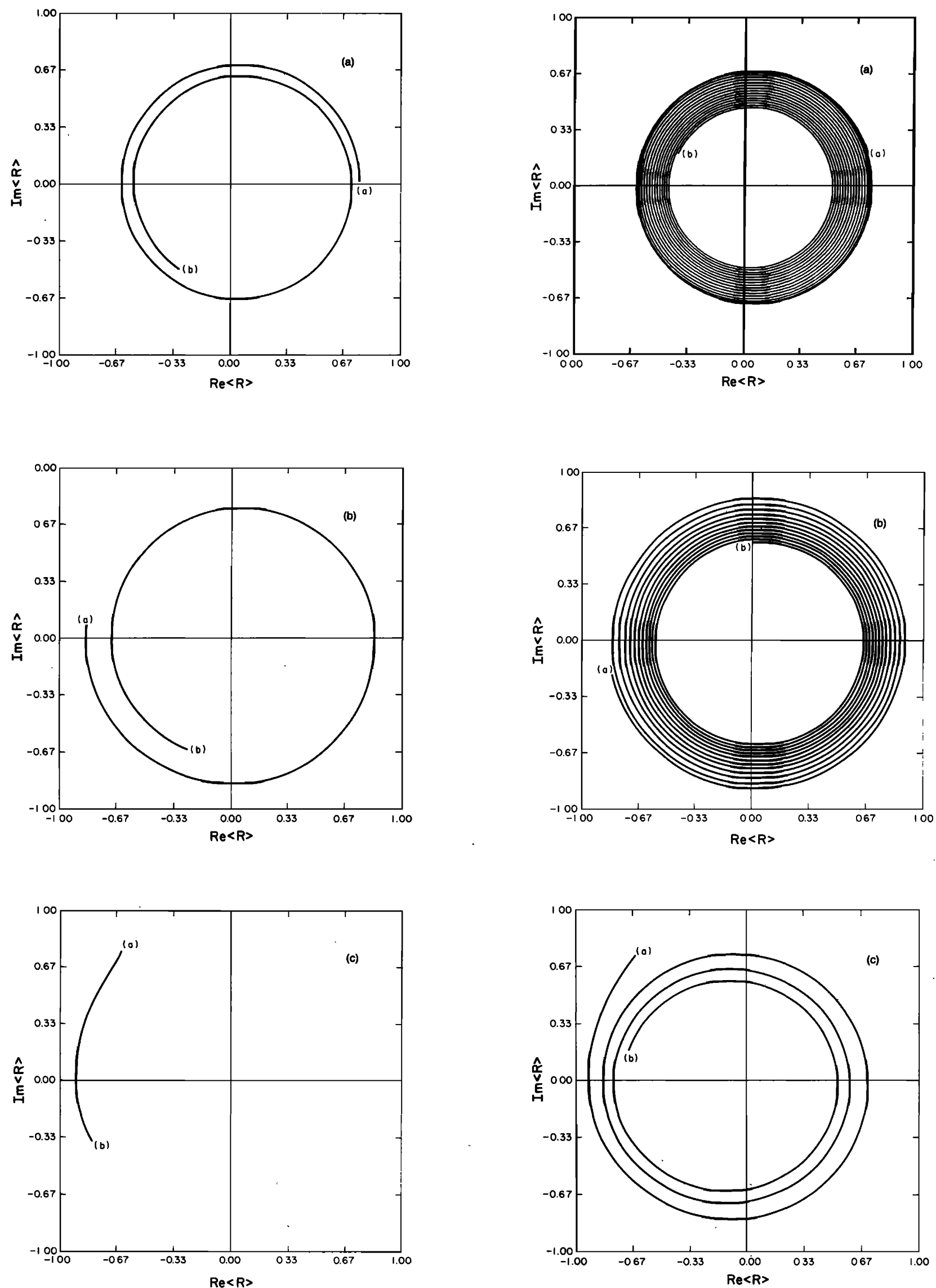

FIG. 2. Variation of mean reflection coefficient with sediment thickness at $25 \mathrm{~Hz}$. (a) $0^{\circ}$ incidence angle, (b) $45^{\circ}$ incidence angle, and (c) $85^{\circ}$ incidence angle. [Points (a) and (b) correspond to thicknesses of 0 and $50 \mathrm{~m}$, respectively.]

FIG. 3. Variation of mean reflection coefficient with sediment thickness at $250 \mathrm{~Hz}$. (a) $0^{\circ}$ incidence angle, (b) $45^{\circ}$ incidence angle, and (c) $85^{\circ}$ incidence angle. [Points (a) and (b) correspond to thicknesses of 0 and $50 \mathrm{~m}$, respectively.] 
(a) $\theta=0$ deg

(b) $\theta=45$ deg

(c) $\theta=85 \mathrm{deg}$

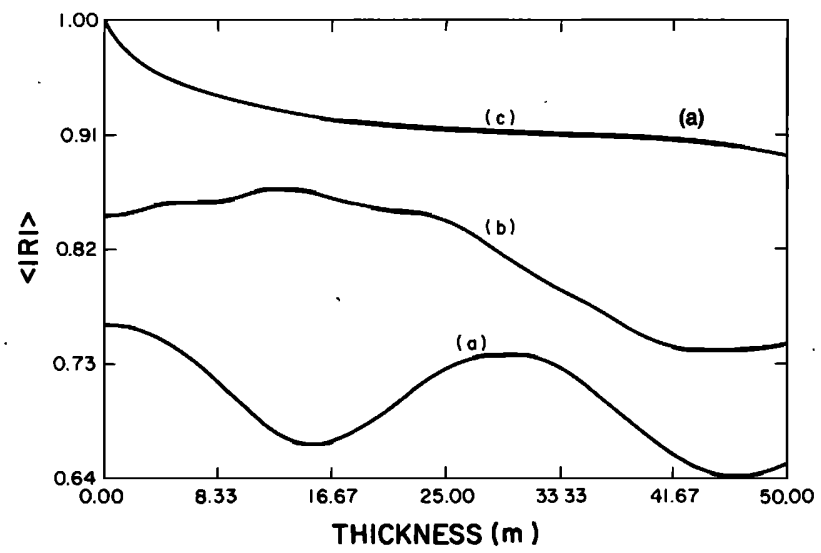

(a) $\theta=0$ deg

(b) $\theta=45 \mathrm{deg}$

(c) $\theta=85 \mathrm{deg}$

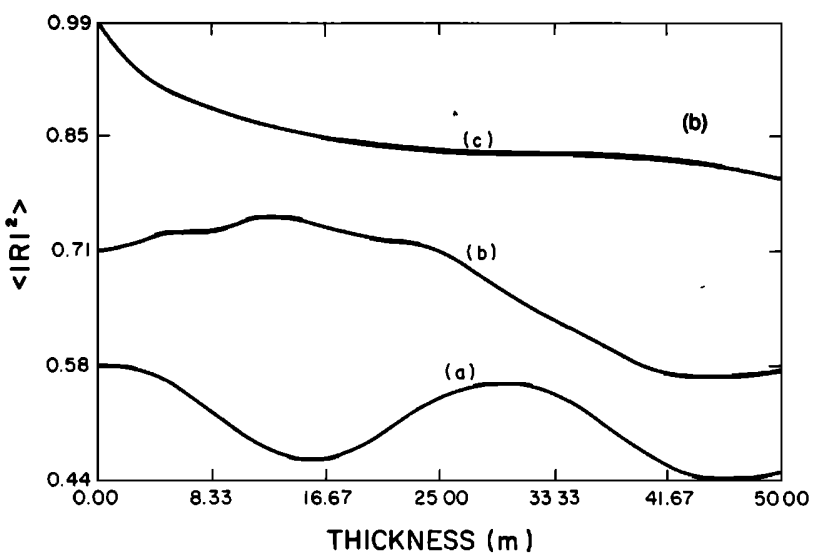

(a) $\theta=0 \mathrm{deg}$

(b) $\theta=45 \mathrm{deg}$

(c) $\theta=85$ deg

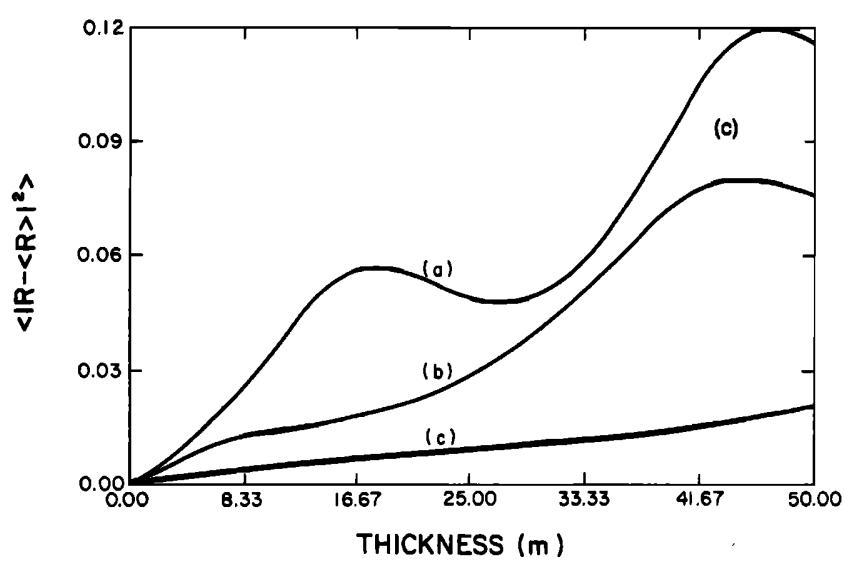

(a) $\theta=0 \mathrm{deg}$

(b) $\theta=45 \mathrm{deg}$

(c) $\theta=85 \mathrm{deg}$

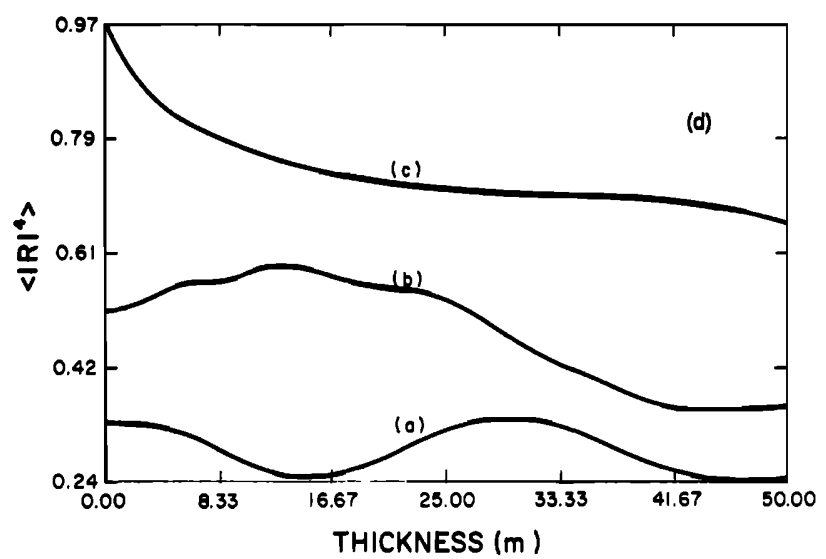

FIG. 4. (a) Mean reflection coefficient modulus versus sediment thickness at $25 \mathrm{~Hz}$. (b) Second moment of reflection coefficient modulus versus sediment thickness at $25 \mathrm{~Hz}$. (c) Centered second moment of reflection coefficient versus sediment thickness at $25 \mathrm{~Hz}$. (d) Fourth moment of reflection coefficient modulus versus sediment thickness at $25 \mathrm{~Hz}$.

the behavior of $E\left\{\left|r e^{i \theta}-E\left\{r e^{i \theta}\right\}\right|^{2}\right\}$; this figure provides added insight into the spreading of the probability mass about its centroid. Viewed collectively, the plots indicate that: (i) at a given sediment thickness, increasing the angle of incidence not only increases the magnitude of the reflection coefficient but also decreases the "spread" in its values; i.e., as incidence angle increases, the center of probability mass migrates toward the perimeter of the unit disk while its moment of inertia simultaneously decreases. Therefore, as incidence angle increases the penetration of acoustic energy into the sediment and the subsequent "randomization" of the returned energy decreases. (ii) At the smaller incidence angles, the moments and their centered moment counterparts undergo a more pronounced, roughly periodic modulation as sediment thickness increases. Since Fig. 2 indicates that the center of probability mass itself spirals smoothly about the origin, this modulation [e.g., of the centered polar moment in Fig. 4(c)] provides a measure of the eccentricity of the probability mass.

Figure 5 exhibits the 250-Hz counterparts to Fig. 4. The same general trends exist in the behavior of the moments, taking into account the frequency scaling. Note, however, that for fixed sediment thickness the moments no longer increase monotonically with incidence angle. Note further that the increase in the spread of the probability mass seems relatively small, given the tenfold increase in frequency. The overall thinness of the layer and the increased dampening effects of dissipation with frequency combine to keep this spreading modest over the frequency range considered.

A numerical implementation of the Trotter approximation formula ${ }^{11}$ was used to solve system (25) and generate the data of Figs. 2-5. Loosely speaking, this formula states that

$\mathbf{p}(l)=e^{-l\left(k \mathscr{L}-Q^{T}\right)} \mathbf{p}(0)=\lim _{n \rightarrow \infty}\left(e^{k(l / n)} e^{(l / n) Q^{T}}\right)^{n} \mathrm{p}(0)$.

Thus, the solution, which involves the simultaneous interplay of advection along characteristics and exchange of probability mass due to the random layering, can be wellapproximated by intertwining the constituent operations. In practice, the limit is replaced by a value of $n$ that is large but finite. This intertwining of an incremental redistribution of probability mass due to random layering followed by an in- 
(a) $\theta=0 \mathrm{deg}$

(b) $\theta=45 \mathrm{deg}$

(c) $\theta=85 \mathrm{deg}$

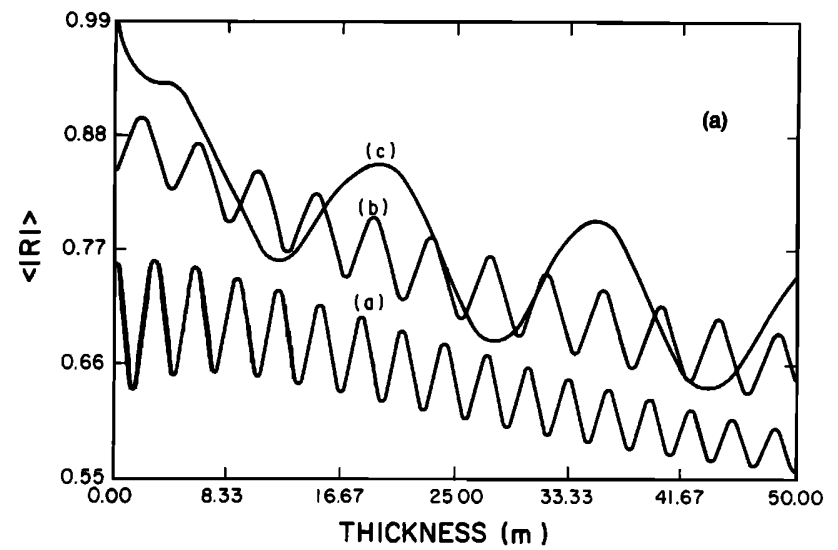

(a) $\theta=0 \mathrm{deg}$

(b) $\theta=45 \mathrm{deg}$

(c) $\theta=85 \mathrm{deg}$

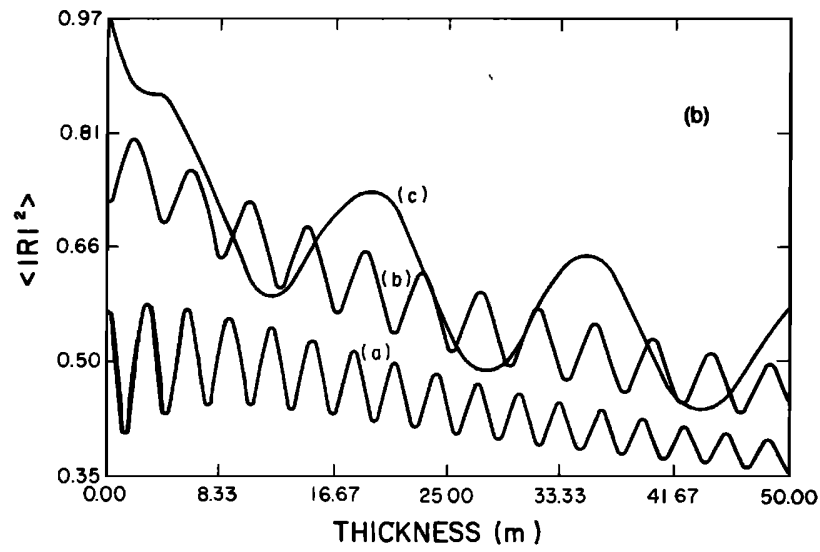

(a) $\theta=0$ dea

(b) $\theta=45 \mathrm{deg}$

(c) $\theta=85 \mathrm{deg}$

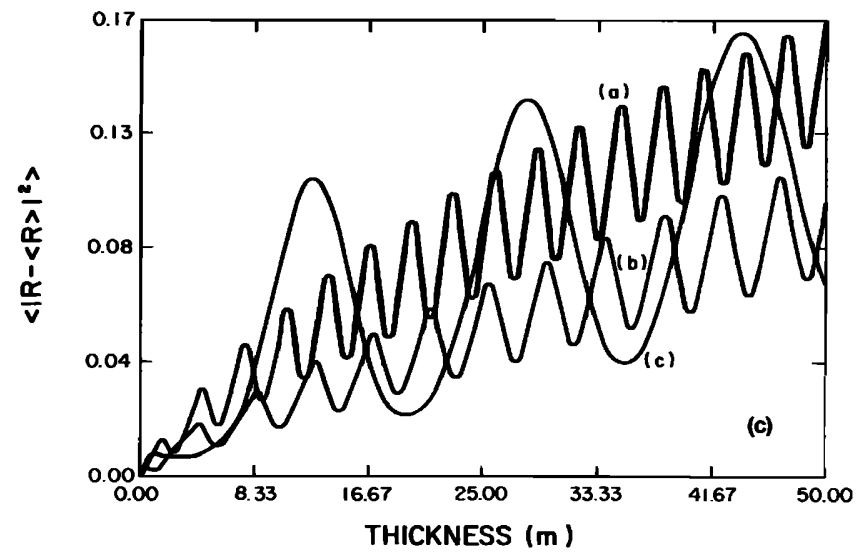

(a) $\theta=0 \mathrm{deg}$

(b) $\theta=45$ deg

(c) $\theta=85 \mathrm{deg}$

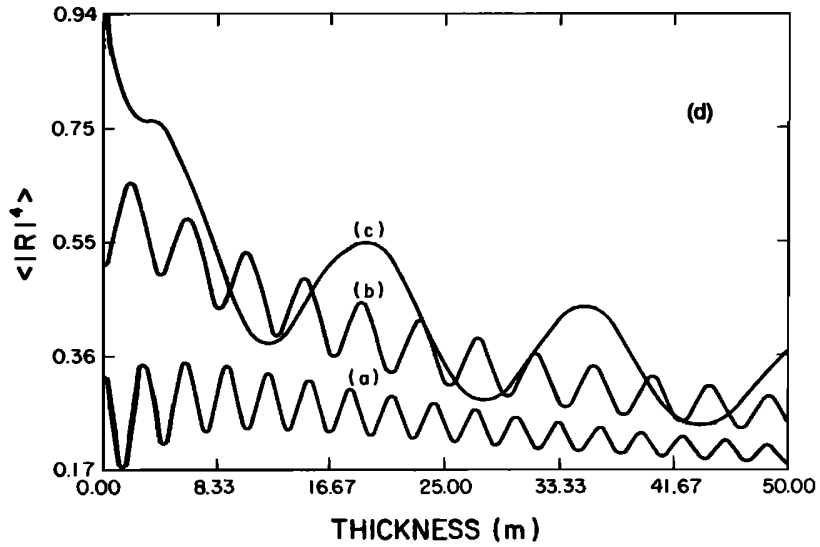

FIG. 5. (a) Mean reflection coefficient modulus versus sediment thickness at $250 \mathrm{~Hz}$. (b) Second moment of reflection coefficient modulus versus sediment thickness at $250 \mathrm{~Hz}$. (c) Centered second moment of reflection coeficient sediment thickness at $250 \mathrm{~Hz}$. (d) Fourth moment of reflection coefficient modulus versus sediment thickness at $250 \mathrm{~Hz}$.

cremental translation along the characteristics (iterated $n$ times) is an approximation that makes sense physically. It is also attractive numerically since the action of each of the semigroups $e^{(l / n) Q^{T}}$ and $e^{-(k l / n) \mathscr{L}}$ is easily determined.

\section{CONCLUSIONS}

A Markov model for the sediment deposition process has been formulated and evolution equations for the probabilistic structure of the interface reflection coefficient have been derived. Within the confines of the model the results are exact. In principle, the extension to multifrequency statistics, i.e., frequency correlations and pulse statistics, is straightforward; in practice, however, the computational demands posed by these problems are formidable.

The analytic structure of the model was used to deduce the appropriate low-frequency limiting smooth geoacoustic model. The interface reflection coefficient statistics of a thin turbidite sediment layer were evaluated numerically at 25 and $250 \mathrm{~Hz}$. In its present state, this one-dimensional Markov model is a highly oversimplified representation of actual ocean sediments, a model that is perhaps best suited for studying the onset of stochastic effects at low frequencies. One potential area for generalization (and added realism), however, is the inclusion of slowly varying lateral variations as a perturbation for which the current laterally homogeneous model would serve as the zeroth-order approximant.

\section{ACKNOWLEDGMENT}

This work was performed under U. S. Office of Naval Research Contract No. N00014-76-C-0056P00004.

\section{APPENDIX: LOW-FREQUENCY ASYMPTOTIC ANALYSIS}

We consider Eq. (25) in the low-frequency regime, where wavelength greatly exceeds average layer thickness. Our goal is to exploit this disparity of scalés in an asymptotic analysis in order to deduce the smooth geoacoustic limit appropriate to our model. Such asymptotic, multiple scale analyses ${ }^{12}$ typically involve the identification of a small parameter and the introduction of a new "slow" variable. The slow-scale dynamics are then determined by the need to suppress secular growth in the higher-order terms of the solu- 
tion expansion. We shall now implement these ideas.

For our problem, wavenumber is an obvious small parameter; let $k \equiv \epsilon<1$. Then (25) becomes

$$
\partial_{\xi} \mathbf{p}+\epsilon \mathscr{L}_{\mathbf{p}}-Q^{\mathbf{T}} \mathbf{p}=\mathbf{0} .
$$

To implement the multiple-scales analysis, we introduce a slow variable $\sigma=\epsilon \xi$ and expand the solution $\mathrm{p}$ as

$$
\mathbf{p}(\xi, \epsilon)=\mathbf{p}_{0}(\xi, \sigma)+\boldsymbol{\epsilon} \mathbf{p}_{1}(\xi, \sigma)+\epsilon^{2} \mathbf{p}_{2}(\xi, \sigma)+\cdots,
$$

where for brevity we suppress the dependence of p upon $(r, \theta)$. Noting that $\partial_{\xi} \rightarrow \partial_{\xi}+\epsilon \partial_{\sigma}$, substitution of (A2) into (A1) leads to the hierarchy of equations:

$$
\begin{aligned}
& \left(\partial_{\xi}-Q^{T}\right) \mathbf{p}_{0}=0, \\
& \left(\partial_{\xi}-Q^{T}\right) \mathbf{p}_{j}=-\left(\partial_{\sigma}+\mathscr{L}\right) \mathbf{p}_{j-1}, \quad j=1,2,3, \ldots
\end{aligned}
$$

From Eqs. (A3) we obtain, in particular,

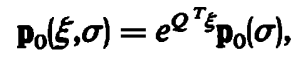

$\mathbf{p}_{0}(\xi, \sigma)=e^{Q^{T_{\xi}} \mathbf{p}_{0}}(\sigma)-\int_{0}^{\xi} e^{\left.Q^{T_{(\xi}}-s\right)}\left(\partial_{\sigma}+\mathscr{L}\right) e^{Q^{T_{s}}} d s \mathbf{p}_{0}(\sigma)$.

We are interested in the solutions (A4) over large distancesdistances that are $O\left(\epsilon^{-1}\right)$ in $\xi$. Since

$$
\begin{aligned}
e^{Q^{T_{\xi}}}= & \left(\left\langle L_{1}\right\rangle+\left\langle L_{2}\right\rangle\right)^{-1} \\
& \times\left[\begin{array}{cc}
\left\langle L_{1}\right\rangle+\left\langle L_{2}\right\rangle e^{-\kappa \xi} & \left\langle L_{1}\right\rangle\left(1-e^{-\kappa \xi}\right) \\
\left\langle L_{2}\right\rangle\left(1-e^{-\kappa \xi}\right) & \left\langle L_{2}\right\rangle+\left\langle L_{1}\right\rangle e^{-\kappa \xi}
\end{array}\right], \\
\kappa \equiv\left\langle L_{1}\right\rangle^{-1}+\left\langle L_{2}\right\rangle^{-1}, &
\end{aligned}
$$

secular growth will occur in $\mathbf{p}_{1}$ (due to the integral term) unless the slow scale evolution of $p_{0}$ is appropriately defined. For brevity, let [cf. (17)]

$$
\begin{aligned}
\mathscr{L}^{(n)} \equiv & \partial_{r}\left\{\left[-\delta^{(n)} r-\frac{1}{2} \delta^{(n)} \cos \theta\left(1+r^{2}\right)\right.\right. \\
& \left.\left.+\frac{1}{2} a_{12}^{(n)}\left(1-r^{2}\right) \sin \theta\right](\cdot)\right\} \\
& +\partial_{\theta}\left\{\left[\frac{1}{2} \delta^{(n)} \sin \theta\left(r^{-1}-r\right)\right.\right. \\
& \left.\left.+\frac{1}{2} a_{12}^{(n)}\left(r^{-1}+r\right) \cos \theta+a_{11}^{(n)}\right](\cdot)\right\}, \quad n=1,2 .
\end{aligned}
$$

Then $\mathscr{L}=\operatorname{diag}\left(\mathscr{L}^{\langle 1\rangle}, \mathscr{L}^{\langle 2\rangle}\right)$ and one can show by direct calculation that

$$
\begin{aligned}
& \int_{0}^{\xi} e^{Q^{T}(\xi-s)}\left(\partial_{\sigma}+\mathscr{L}\right) e^{Q^{T_{s}}} d s \mathbf{p}_{0}(\sigma) \\
& \sim \xi\left[\partial_{\sigma}+\left(\left\langle L_{1}\right\rangle+\left\langle L_{2}\right\rangle\right)^{-1}\left(\left\langle L_{1}\right\rangle \mathscr{L}^{(1\rangle}+\left\langle L_{2}\right\rangle \mathscr{L}^{(2)}\right)\right] \\
& \quad \times\left(\left\langle L_{1}\right\rangle+\left\langle L_{2}\right\rangle\right)^{-1}\left[\begin{array}{ll}
\left\langle L_{1}\right\rangle & \left\langle L_{1}\right\rangle \\
\left\langle L_{2}\right\rangle & \left\langle L_{2}\right\rangle
\end{array}\right] p_{0}(\sigma), \quad \xi \rightarrow \infty .
\end{aligned}
$$

The asymptotic dynamics of (27) follow by requiring that secular term (A7) vanish. Note that $\sigma=\epsilon \xi=k \xi$ and that the scalar function $p_{0}$ in (27) is simply the sum of the two components of $\mathbf{p}_{0}$ in (A7).

${ }^{1}$ K. E. Gilbert, J. Acoust. Soc. Am. 68, 1454-1458 (1980).

${ }^{2}$ A. Papoulis, Probability, Random Variables and Stochastic Processes (McGraw-Hill, New York, 1965).

3J. A. Morrison, J. Math. Phys. 13, 299-306 (1972).

${ }^{4}$ L. M. Brekhovskikh, Waves in Layered Media (Academic, New York, 1960).

${ }^{5} \tau$, being a stochastic process, is actually a function of two variables, $\xi$ and a probability space variable which we shall call $\alpha$. For every fixed $\xi, \tau \xi, \cdot)$ is a random variable; for every fixed $\alpha, \tau(\cdot, \alpha)$ is a sample function or realization which in this case will be a piecewise constant function assuming $N$ integer values. For brevity, we do not explicitly indicate the probability space variable in the notation.

${ }^{6} o(\delta \xi)$ refers to terms which tend to zero faster than $\delta \xi$ itself as $\delta \xi \rightarrow 0$. A definition of the order symbols can be found, for example, in the text: $\mathbf{N}$. Bleistein and R. Handelsman, Asymptotic Evaluation of Integrals (Holt, Rinehart and Winston, New York, 1975).

${ }^{7}$ H. E. Morris, J. Acoust. Soc. Am. 48, 1198-1202 (1970).

8J. L. Doob, Stochastic Processes (Wiley, New York, 1953), pp. 243-244.

${ }^{9}$ M. Schoenberg and P. N. Sen, J. Acoust. Soc. Am. 73, 61-67 (1983).

${ }^{10}$ K. E. Gilbert, J. Acoust. Soc. Am. 73, 137-142 (1983).

${ }^{11}$ H. F. Trotter, Proc. Am. Math. Soc. 10, 545-551 (1959).

${ }^{12} \mathrm{~J}$. Kervorkian and J. D. Cole, Perturbation Methods in Applied Mathemat ics (Springer-Verlag, New York, 1981). 